\title{
In Situ Ferroelectric Domain Dynamics Probed with Differential Phase Contrast Imaging
}

\author{
Abinash Kumar ${ }^{1}$, Rohan Dhall ${ }^{2}$ and James M. LeBeau ${ }^{1 *}$ \\ 1. Department of Materials Science and Engineering, North Carolina State University, Raleigh, NC, \\ USA \\ 2. National Center for Electron Microscopy, Molecular Foundry, Lawrence Berkeley National \\ Laboratory, Berkeley, CA, USA \\ * Corresponding author: lebeau@mit.edu
}

Unlike classical ferroelectrics, relaxor ferroelectrics are characterized by their diffuse phase transitions and dielectric properties. They can also exhibit a low coercive field and giant piezoelectric coefficients [1]. These properties are thought to arise from the existence of polar nanoregions in a non-polar matrix or a slush-like response of a nanoscale multi-domain state, but the models are fully predictive [2,3]. If relaxor ferroelectric materials are to be designed rather than discovered, a complete fundamental understanding of their response to electric field is required - from the micro to atomic length scale. Recent advances in in situ microscopy and STEM detector technologies, such as 4D STEM and differential phase contrast (DPC) imaging [4], have the potential to unlock the structural details needed to explain the remaining mysteries in these materials.

In this presentation, we will show how DPC provides robust imaging of the relaxor ferroelectric domain structure as a function of bias, regardless of sample tilt. We apply the technique to $\mathrm{Pb}\left(\mathrm{Mg}_{1 / 3} \mathrm{Nb}_{2 / 3}\right) \mathrm{O}_{3-}$ $28 \mathrm{PbTiO}_{3}$ (PMN-PT) single crystals prepared using conventional polishing methods pair with photolithography to define the electrodes. First, we will discuss how the electron beam influences the domain structure, which is studied by examining a series of DPC images without an applied field, as shown in Figure 1a. The electron beam causes the domain walls to flicker in the time-resolved dataset (see arrow), but large-scale changes are not observed at this field of view. We will also show the dramatic change in domains at different applied electric fields, Figure 1b. The domain structure at the start and end of a field cycle is explained in the context of remnant polarization. To determine how the waveform shape affects switching, we will also discuss results comparing electric fields applied using sawtooth or square waves. For the square wave, nanoscale lamellar domains are found to form when the electric field amplitude switches between $\pm 9 \mathrm{kV} / \mathrm{cm}$, but are not observed when switching between $\pm 54 \mathrm{kV} / \mathrm{cm}$.

To quantify changes at the atomic scale, we will demonstrate how in situ integrated differential phase contrast (iDPC) provides high signal-to-noise ratio imaging of oxygen atom columns, even in the presence of heavier atoms $(\mathrm{Pb})$. Further, we will also show how the simultaneous acquisition of annular dark field (ADF) can be used to simplify the quantification and removal of drift and scan distortion from iDPC images [5]. From the drift-corrected iDPC images, the projected polarization is then quantified for same atom columns at different applied fields. As shown in Figure 2, the polar nanoregions try to align to the electric field, but reside within a microscale ferroelectric domain that does not switch. Equipped with a combination of data across length scales for the same sample, we will discuss how the resulting details provide new insights into the dynamics of relaxor ferroelectric domains [6].

\section{References:}

[1] F. Li et al., Nature Materials 17 (2018), p. 349.

[2] F. Li et al., Nature Communications 7 (2016), p. 13807. 
[3] H. Takenaka et al., Nature 546 (2017), p.391.

[4] N. Shibata et al., Nature Physics 8 (2012), p. 611.

[5] X. Sang and J. M. LeBeau, Ultramicroscopy 138 (2014), p.28.

[6] This material is based upon work supported by the National Science Foundation, as part of the Center for Dielectrics and Piezoelectrics under Grant Nos. IIP-1361571 and IIP-1361503.
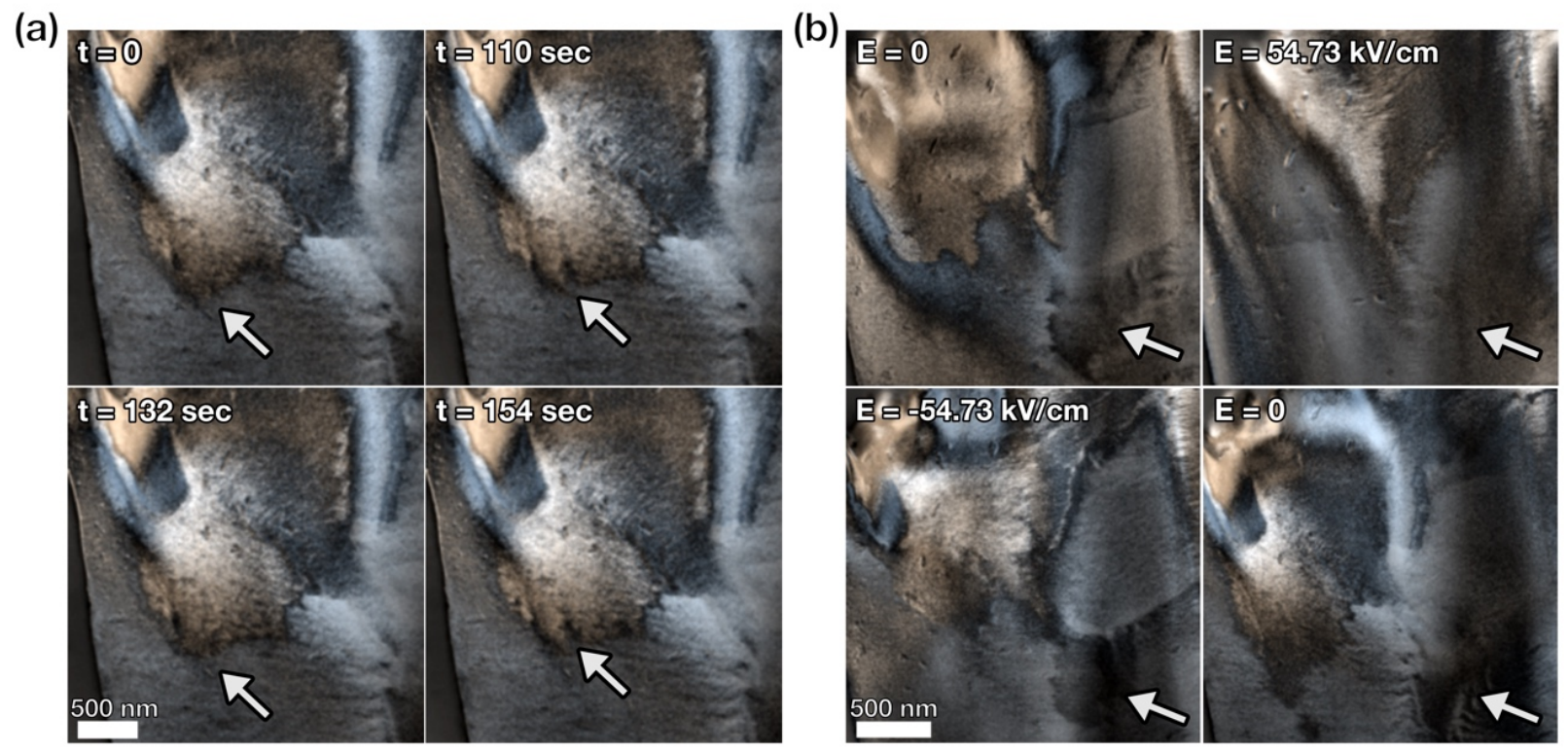

Figure 1. DPC images of PMN-PT (a) exposed to the electron beam without an applied electric field, and (b) at the electric fields indicated.
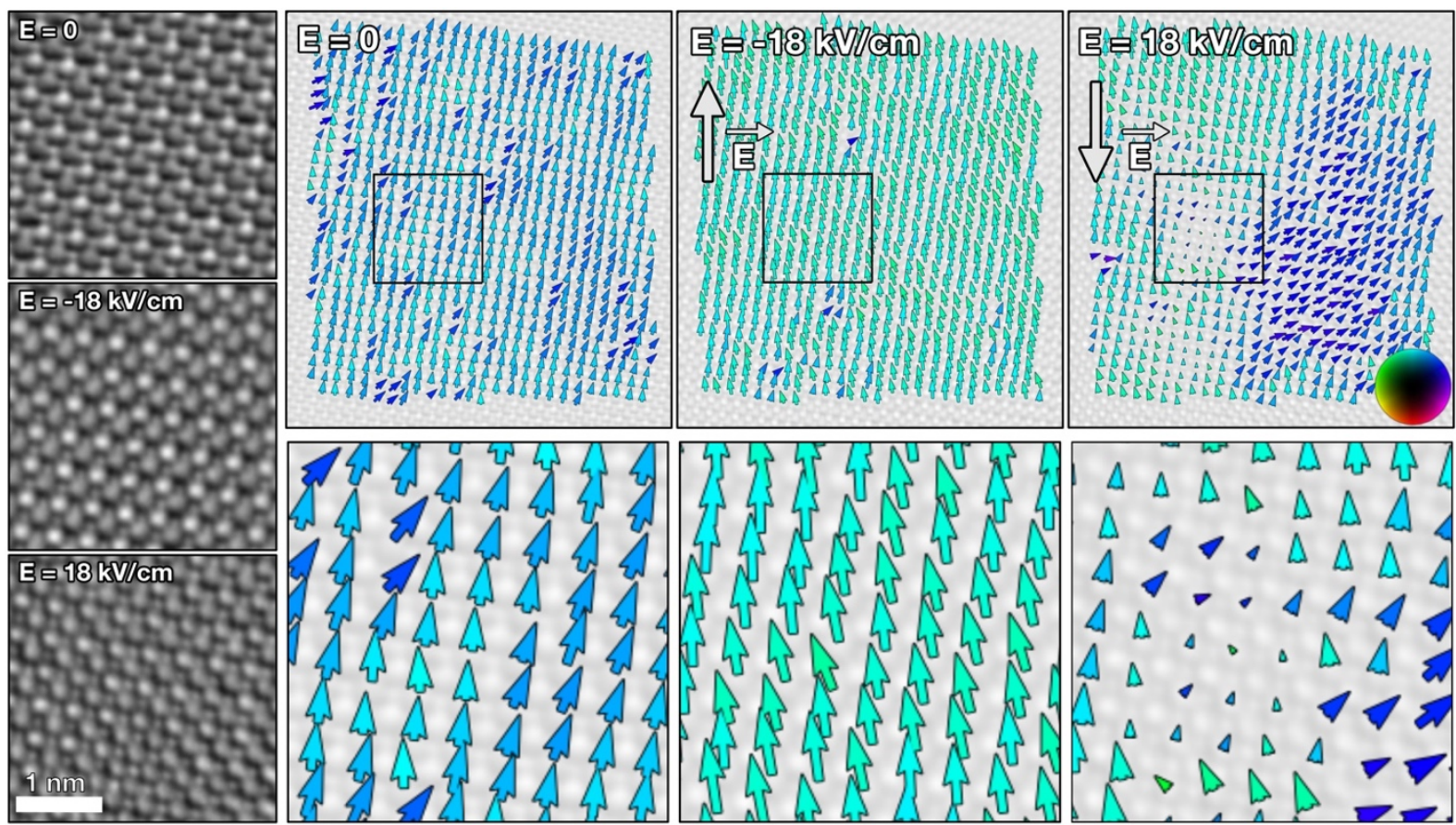

Figure 2. (left) iDPC images from the square regions marked in the polarization maps. Projected polarization is determined from both the cation and anion positions for the electric fields indicated. 